\title{
Orthopaedic management in extreme genu valgum and recurvatum in a patient with rheumatoid arthritis
}

\author{
Calin Dragosloveanu', Serban Dragosloveanu', Bogdan Cretu', Ioana Cretu \\ ${ }^{1}$ Clinical Hospital for Orthopedics-Traumatology and osteoarticular TB Foisor, Bucharest, Romania \\ 2"Dr. I.Cantacuzino" Clinical Hospital, Bucharest, Romania
}

\begin{abstract}
A case of acquired severe genu valgum and genu recurvatum in a patient with rheumatoid arthritis (RA) is here reported. The pain and genu valgum progressed because of poor RA control. The patient had no history of major trauma of the knee before or after the onset of RA. The most reasonable hypothesis to explain this patient's pathology is that occult genu recurvatum developed after a minor trauma and progressed; poor RA control then worsened the stability, the osteopenia and genu valgum, in the end the patient acquired a severe genu valgum with extreme values of the tibiofemoral axes (TFM), with multiplanar knee laxity and important bone destruction. Total knee arthroplasty (TKA) was successfully performed using a constrained hinged prosthesis. One year after the surgery, the patient exhibited improvement in her Knee Society Score from preoperative scores of 19 to postoperative scores of 91 , no early or late signs of infection occurred, no early signs of loosening were noted either. Acquired global ligamentous deficiency with hyperextension instability (genu recurvatum) associated with severe genu valgum in patients with RA is rare. Excellent results were obtained with TKA, but the use of the most constrained prosthesis due to massive bone loss and ligamentous deficiency comes with the disadvantages of early aseptic loosening as a result of increased constrained compared to an unconstrained design.
\end{abstract}

Keyword: genu valgum; genu recurvatum; total knee arthroplasty; rheumatoid arthritis

\section{INTRODUCTION}

Total knee arthroplasty (TKA) in rheumatoid arthritis (RA) is difficult to manage because of the unique particularities of the disease. These include ipsilateral hip involvement, anticoagulation needs, flexion contractures, rheumatoid cysts and the need for synovectomy (1). Patients with RA are at high risk of early and late infections. Osteopenia can lead to intraoperative and postoperative fractures (2). Bone deficiencies are often important mainly in the posterior aspect of the tibia and they need to be restored for proper position of the prosthesis (3). Hyperextension instability is a rare condition in patients with RA and it is usually secondary to knee trauma. Genu valgum combined with genu recurvatum and multiplanar laxity leads to rapid progression of the gonarthrosis witch becomes painful and debilitating.

Rheumatoid arthritis is a chronic inflammatory disease associated with systemic immunologic ab- normalities. RA mainly affects the synovial joints within the upper and lower extremities, and it may result in severe damage or destruction of the affected joints although genu valgum of the lower extremities is often seen in patients with RA, acquired genu recurvatum is very rare (4). We herein present a case of acquired genu recurvatum associated with severe genu valgum in a patient with RA that was successfully treated with TKA.

\section{CASE REPORT}

A 68-year-old woman developed an acquired hyperextension instability associated with severe genu valgum in her left knee. She has been diagnosed with RA at the age of 51 years. She had no complaints regarding her left knee before the onset of RA. Previous treatments between the age of 51 and the age of 60 included only pain management treatment and local corticosteroids done by the general 
practitioner, no disease modifying antirheumatic drugs (DMARD's) was administered in this interval. In this period we have no radiological data to analyze the progression of the gonarthrosis. Her most recent treatment was methotrexate at $15 \mathrm{mg} /$ week and leflunomide $20 \mathrm{mg} /$ day for the last 2 years. Her preoperative RA status was classified as stage IV, class III (5). Laboratory test results showed a C-reactive protein (CRP) level of $0.67 \mathrm{mg} / \mathrm{dL}$, erythrocyte sedimentation rate (ESR) between normal values and rheumatoid factor positivity $(42 \mathrm{IU} / \mathrm{mL})$. Her preoperative Disease Activity Score and Modified Health Assessment Questionnaire score were 2.81 and 1.625 , respectively $(6,7,8)$.

According to the patient, the genu valgum began to progress at the age of 59 years and at the age of 62 the patient suffered a mild trauma in her left knee and started to accuse instability in her knee since then. Because no data on the tender and swollen joint counts were obtained from the medical record before the surgery, the disease activity at that time was estimated according to the CRP. From the age of 51 to the age of 60 years, the patient's RA activity was poorly controlled. From the age of 60 to 68 years, her RA activity became better controlled; the mean CRP levels were between $0.30 \mathrm{mg} / \mathrm{dL}$ and 0.60 $\mathrm{mg} / \mathrm{dL}$. The pain in her left knee was aggravated while weight bearing at the age of 62 years, at which time she suffered the mild trauma. However, the patient did not remember the onset of the hyperextension, nor could it be estimated according to the clinical course.

Physical examination revealed a hyperextension of $20^{\circ}$, a flexion of $60^{\circ}$ and global laxity and instability. Despite the extreme genu valgum the patellar

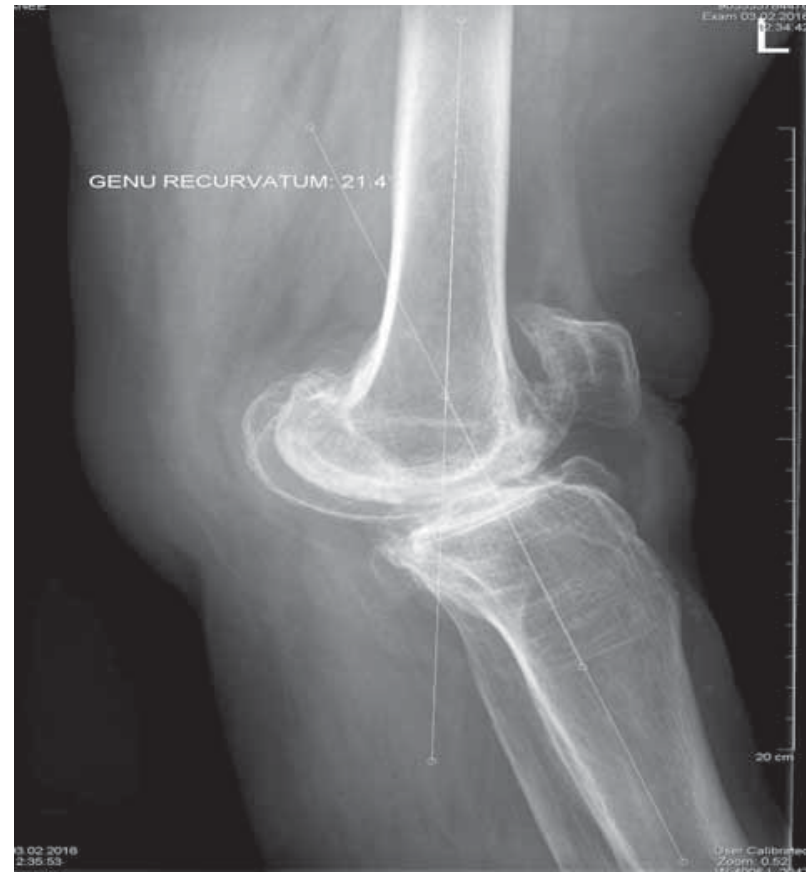

FIGURE 1

tracking was normal. The patient could walk a maximum distance of $30 \mathrm{~m}$. Her preoperative Knee Society Scores was 16 (9). Radiographic examination revealed severe genu valgum of the left lower limb with a tibiofemoral axes (TFM) of 148.1 and hipknee-ankle (HKA) angle of $29.7^{\circ}$ valgus in the weight bearing position. This severe genu valgum was uncorrectable by manual stress. The lateral incidence in weight bearing position showed $21.4^{\circ} \mathrm{de}-$ grees of hyperextension (Fig. 1). Loss of the joint space at the lateral femorotibial and patellofemoral compartment was recognized. The other large joints of the lower limbs (bilateral hips, right knee, and bilateral ankles) were mostly normal, and no systemic joint laxity was observed.

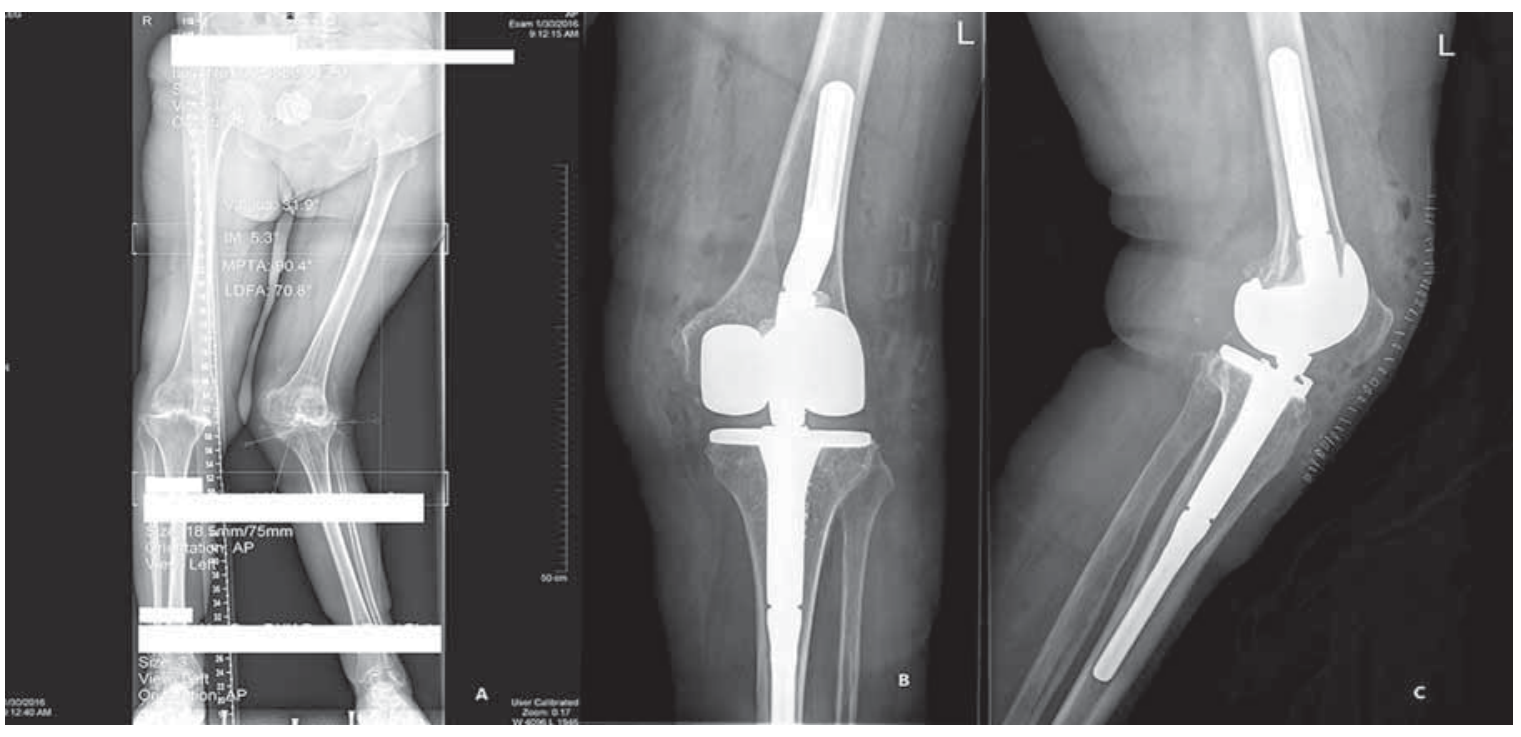


TKA was planned because of the severity of the pain and deformity. We routinely establish a preoperative plan for TKA operations using a conventional two-dimensional template with X-ray films, long leg weight bearing anteroposterior (AP) view and lateral view. From the beginning the surgeon decided to use a constrained hinged prosthesis, because of the global laxity and important bone loss. On the AP view we measured the TFM angle, the hip-knee ankle angle (HKA), the lateral distal femoral angle (LDFA) and the medial proximal tibial angle (MPTA) (Fig. 2).

The surgeon used a mini invasive approach, a subvastus approach (Fig. 3). The lateral femorotibial and patellofemoral compartments had abundant synovium and severe bone loss. Routine exposure proceeds with partial excision of the Hoffa fat pad, excision of the anterior horns of the meniscus, resection of the cruciate ligaments and removal of the peripheral femoral and tibial osteophytes. In this case particularly the cruciate ligaments and the medial collateral ligament were already completely torn (10). After these primary steps the surgeon resected the distal femur and the proximal tibia according to the technique. After those preliminary cuts the balancing of the extension gap by a lateral release (pie crusting release) was done. Next, the femur and tibia were cut via the subvastus approach according to the recommendations of the manufacturer. The femoral component was placed parallel to the surgical epicondylar axis and in $3^{\circ}$ of external rotation from the

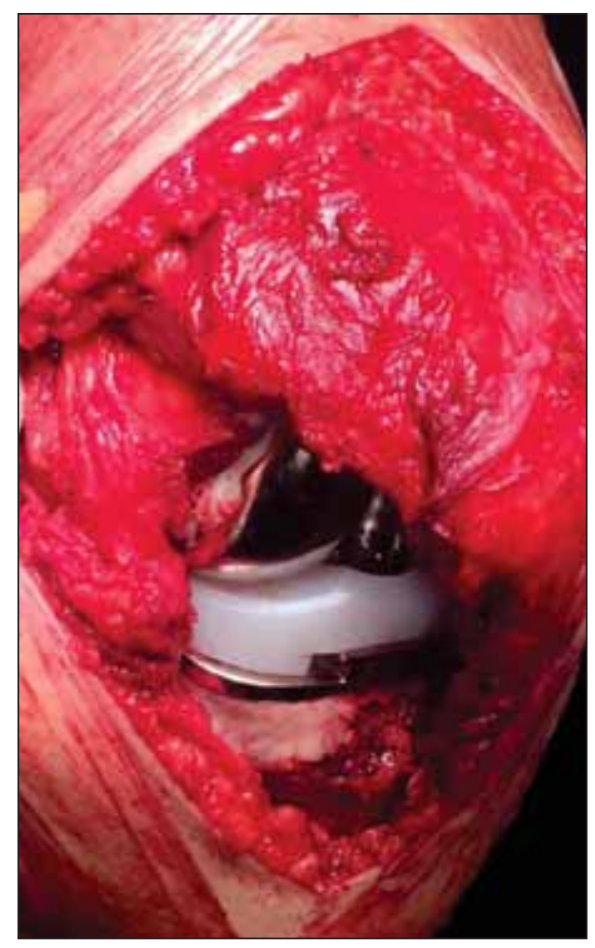

FIGURE 3 posterior epicondyles, and the center of the tibial component was aligned in relation to the tibial tuberosity with regard to rotation and translation. Finally, the knee was replaced with a constrained hinged prosthesis (RHK; Zimmer, Warsaw, IN, USA) to provide security against the global instability (Fig. 2). Upon completion of the implantation, the patella was confirmed to track centrally within the trochlear groove of the femoral component through the full ROM.

Active and passive motion and a standing gait exercise program were started on postoperative day (POD) 2 following the same rehabilitation protocol for standard TKA. On POD 21, the patient was able to walk without assistance, and the ROM of the operated knee was from $0^{\circ}$ to $90^{\circ}$. She was discharged on POD 5 with normal wound healing and no other complications. At the 6 weeks, 3 months, 6 months and 1 year postoperative follow-up evaluations, the knee was stable and pain-free. Good patella tracking was confirmed, and the ROM was from $0^{\circ}$ to $100^{\circ}$. Walking was unlimited without assistance, and the Knee Score at 6 months was 94. The patient stated that her left knee was excellent and that she was fully satisfied with the surgery. Her lower limb alignment was also excellent with an TFM angle of $174^{\circ}$ and an HKA angle of $2.5^{\circ}$ valgus on the AP full leg length weight-bearing X-ray. All component positions were excellent according to the Knee Society Radiographic Evaluation Form (11). No evidence of loosening or patellar dislocation was present at 1 year. No early or late signs of infection were seen.

\section{DISCUSSION}

Although genu valgum of the lower extremities is often seen in patients with RA, acquired hyperextension (genu recurvatum) after onset of RA is quite rare. Although acquired hyperextension deformity is usually secondary to trauma, this patient had no history of major trauma to the left knee either before or after the onset of RA. The pain aggravated in her left knee at the age of 62 years right after the mild trauma, we can imply that this was the moment when the patient torn her cruciate ligaments and with pre-existing background of genu valgum and other knee lesions specific to RA conducted to extreme deviations, global instability and important bone loss. Essentially to this case is the fact that the patient did not attend any rheumatologist and did not adhere to any targeted treatment between the age of 51 and 60 
years old. In this period the poor RA control induced progression to the gonarthrosis.

It is difficult to conceive that only genu valgum with inflammatory soft tissue damage was responsible for the acquired genu recurvatum in this case. Instead, a combination of internal and external factors is considered to have contributed to the etiology of the patellar dislocation. First, RA control was poor for 9 years after the patient began to feel mild pain in her left knee at the age of 51 years; in this period inflammatory damage to the surrounding soft tissue was probably done. Second, a minor trauma with a pre-existing background of slight genu valgum caused cruciate ligaments complete rupture and progression of the deformity.
Our patient achieved good knee function in the third postoperative week, and this function was retained at the 1-year postoperative follow-up. X-ray evaluation of the lower limb alignment and patellofemoral joint showed excellent results.

In conclusion, we have reported a rare case of acquired genu recurvatum associated with severe genu valgum in a patient with RA. TKA with constrained hinged prosthesis provided excellent results, but the use of the most constrained prosthesis comes with the disadvantages of early aseptic loosening as a result of increased constrained compared to an unconstrained design.

Conflict of interest: none declared Financial support: none declared

\section{REFERENCES}

1. Goldring S.R., Wojno W.C., Schiller A.L. et al. In patients with rheumatoid arthritis the tissue reaction associated with loosened total knee replacements exhibits features of a rheumatoid synovium. J Orthop Rheum 1:9-21, 1988.

2. Aaron R.K., Scott R.D. Supracondylar fracture of the femur after total knee arthroplasty. Clin Orthop 219:136-139, 1987.

3. Laskin R.S. Total condylar knee replacement in patients who have rheumatoid arthritis: a ten-year follow-up study. J Bone Joint Surg Am 72:529-535, 1990.

4. Mochizuki T., Saito S. Total knee arthroplasty for massive joint destruction in a patient with rheumatoid arthritis complicated with fibrous dysplasia. Mod Rheumatol. 2009;19:204e208.

5. Steinblocker O., Traeger C.H., Batterman R.C. Therapeutic criteria in rheumatoid arthritis. J Am Med Assoc. 1949;140:659e662.

6. Prevoo M.L., van't Hof M.A., Kuper H.H., van Leeuwen M.A., van de Putte L.B., van Riel P.L. Modified disease activity scores that include twenty-eight-joint counts. Development and validation in a prospective longitudinal study of patients with rheumatoid arthritis. Arthritis Rheum. 1995;38:44e48.
7. Inoue E., Yamanaka H., Hara M., Tomatsu T., Kamatani N. Comparison of disease activity score (DAS) 28- erythrocyte sedimentation rate and DAS28-C-reactive protein threshold values. Ann Rheum Dis. 2007:66:407e409.

8. Pincus T., Summey J.A., Soraci Jr. S.A., Wallston K.A., Hummon N.P. Assessment of patient satisfaction in activities of daily living using a modified Stanford Health Assessment Questionnaire. Arthritis Rheum. 1983;26:1346e1353.

9. Insall J.N., Dorr L.D., Scott R.D., Scott W.N. Rationale of the knee society clinical rating system. Clin Orthop. 1989;248:13e14.

10. Dragosloveanu S. et al. Outcome of iatrogenic collateral ligaments injuries during total knee arthroplasty. Eur J Orthop Surg Traumatol. 2014; 24(8):1499-503.

11. Ewald F.C., on behalf of the Knee Society. The knee society total knee arthroplasty roentgenographic evaluation and scoring system. Clin Orthop. 1989; 248:9e12. 